\title{
AN EFFICIENT METHOD FOR BAND STRUCTURE CALCULATIONS IN 3D PHOTONIC CRYSTALS
}

\author{
DAVID C. DOBSON, JAYADEEP GOPALAKRISHNAN, AND JOSEPH E. PASCIAK
}

\begin{abstract}
A method for computing band structures for three-dimensional photonic crystals is described. The method combines a mixed finite element discretization on a uniform grid with a fast Fourier transform preconditioner and a preconditioned subspace iteration algorithm. Numerical examples illustrating the behavior of the method are presented.
\end{abstract}

\section{IntRODUCTION}

Photonic crystals are structures constructed of dielectric materials arranged in a periodic array. In this paper we consider structures which are periodic along each of the three orthogonal coordinate axes in space. Such structures have been found to exhibit interesting spectral properties with respect to classical electromagnetic wave propagation, including the appearance of band gaps. Three-dimensional photonic band gap structures have many possible applications in lasers, microwaves, optical communications, etc. See $[4,14]$ for more information on photonic crystals and their applications.

Since fabrication of these structures is currently quite challenging, computation has become the primary tool for investigating the spectra of photonic crystals. In this paper we propose a new computational method based on a mixed finite element discretization of Maxwell's equations, combined with a fast Fourier transform (FFT) preconditioner and a preconditioned subspace iteration algorithm for finding eigenvalues. Finite element methods have already been introduced for $2 \mathrm{D}$ photonic crystals $[1,8]$. The $2 \mathrm{D}$ case is much simpler than the $3 \mathrm{D}$ case since the underlying problem is scalar, and a classical finite element discretization can be used. For simplicity we consider here only the simple cubic (sc) lattice geometry. Except for the FFT preconditioner, the same basic techniques extend to other typical lattice geometries.

Several other techniques have been developed for band structure calculations in general 3D photonic crystals (see for example the survey [4] and the references therein; see also [20]). The most popular methods are based on truncated plane wave decompositions of the electromagnetic field $[12,25]$. These approaches are very natural and can offer fast convergence in situations where the spatial variation of the medium is smooth. In photonic crystals however, it is much more common that the underlying medium is discontinuous. In this case Gibbs-type phenomena may lead to slow convergence of the truncated field [25]. In this paper we develop a method which is naturally suited to handle discontinuous media, using finite element approximations which conform to the material interfaces and do not suffer from Gibbs phenomena.

In the next section, we formulate the underlying eigenvalue problem in mixed form over appropriate function spaces. In section 3, we then develop a discretization scheme

This research was supported by NSF grant number DMS-9626567, AFOSR grant number F4962098-1-0005 and Alfred P. Sloan Research Fellowship. 
for the mixed problem using Nedelec edge elements specially modified to give stable approximations. In section 4, we construct a fast Fourier transform preconditioner to be used in an iterative eigenvalue approximation algorithm described in section 5. Finally in section 6, the results of several numerical experiments are described.

\section{Problem Formulation}

We consider classical electromagnetic wave propagation governed by Maxwell's equations

$$
\begin{aligned}
& \nabla \times E-i \omega \mu H=0, \\
& \nabla \times H+i \omega \epsilon E=0,
\end{aligned}
$$

where the magnetic permeability $\mu$ is assumed constant, and the dielectric coefficient $\epsilon$ is real, bounded and uniformly bounded away from zero. The above equations hold on all of $\mathbb{R}^{3}$. Setting $\gamma=(\mu \epsilon)^{-1}$, it follows from $(1,2)$ that

$$
\begin{aligned}
\nabla \times \gamma \nabla \times H & =\omega^{2} H, \quad \text { on } \mathbb{R}^{3}, \\
\nabla \cdot H & =0, \quad \text { on } \mathbb{R}^{3} .
\end{aligned}
$$

The medium is assumed to have unit periodicity on a cubic lattice. Thus denoting $Z=\{0, \pm 1, \pm 2, \ldots\}$, and defining the lattice $\Lambda=Z^{3}$, we have

$$
\epsilon(x+n)=\epsilon(x), \quad \text { for all } x \in \mathbb{R}^{3} \text {, and for all } n \in \Lambda \text {. }
$$

We define the periodic domain $\Omega=\mathbb{R}^{3} / \Lambda$ and the first Brillouin zone $K=[-\pi, \pi]^{3}$.

We are interested in finding Bloch eigenfunctions [22], that is, functions $H$ satisfying $(3,4)$ for a particular frequency $\omega$, and such that $H(x)=e^{i \alpha \cdot x} H_{\alpha}(x)$, where $H_{\alpha}$ is periodic in $x$, and $\alpha \in K$. For each $\alpha \in K$, it then follows from $(3,4)$ that

$$
\begin{aligned}
\nabla_{\alpha} \times \gamma \nabla_{\alpha} \times H_{\alpha} & =\omega^{2} H_{\alpha}, \quad \text { in } \Omega, \\
\nabla_{\alpha} \cdot H_{\alpha} & =0, \quad \text { in } \Omega,
\end{aligned}
$$

where $\nabla_{\alpha}=(\nabla+i \alpha)$. We will henceforth consider only the transformed system $(5,6)$ and so to simplify notation we drop the subscript $\alpha$ when referring to $H_{\alpha}$.

We get a weak formulation of $(5,6)$ by introducing the spaces

$$
\begin{aligned}
V & \equiv H(\text { curl })=\left\{F \in L^{2}(\Omega)^{3}: F \text { periodic and } \nabla \times F \in\left(L^{2}(\Omega)\right)^{3}\right\}, \\
V^{0, \alpha} & =\left\{F \in V: \nabla_{\alpha} \cdot F=0 \text { in } \Omega\right\} .
\end{aligned}
$$

In the above definitions, the differentiation is defined in the distributional sense. For $F, G \in V$, we introduce the hermitian forms

$$
\begin{aligned}
& a(F, G)=\int_{\Omega} \gamma\left(\nabla_{\alpha} \times F\right) \cdot \overline{\left(\nabla_{\alpha} \times G\right)} d x, \\
& c(F, G)=\int_{\Omega} F \cdot \bar{G} d x .
\end{aligned}
$$

The weak formulation of $(5,6)$ is then to find $\omega \in \mathbb{R}$ and $H \in V^{0, \alpha}$ satisfying

$$
a(H, F)=\omega^{2} c(H, F) \quad \text { for all } F \in V^{0, \alpha} .
$$

The form $a(\cdot, \cdot)$ is positive semidefinite on $V \times V$ and is positive definite on $V^{0, \alpha} \times V^{0, \alpha}$ for $\alpha \neq(0,0,0)$. Of course, $c(\cdot, \cdot)$ is positive definite on $V \times V$. 
We will consider (9) in mixed form. Let $W \equiv H^{1}=\left\{g \in L^{2}(\Omega): g\right.$ periodic and $\nabla g \in$ $\left.\left(L^{2}(\Omega)\right)^{3}\right\}$ and for $\rho \in H^{1}$ and $F \in V$, define

$$
b(\rho, F)=\int_{\Omega} \nabla_{\alpha} \rho \cdot \bar{F} d x .
$$

The mixed form of (9) is then: find $\omega \in \mathbb{R}$ and $(H, \rho) \in V \times W$ such that

$$
\begin{aligned}
a(H, F)+b(\rho, F) & =\omega^{2} c(H, F), \quad \text { for all } F \in V, \\
\overline{b(g, H)} & =0, \quad \text { for all } g \in W .
\end{aligned}
$$

The stability of the mixed reformulation depends two conditions [7]. The first is the so-called Ladyzhenskaya-Babuška-Brezzi (LBB) condition $[16,2,6]$, i.e., there is a constant $C$ such that

$$
\|w\|_{W} \leq C \sup _{\substack{X \in V \\ X \neq 0}} \frac{|b(w, X)|}{\|X\|_{V}},
$$

for all $w \in W$. The norms above are the natural norms in $V$ and $W$. The second condition is that $a(\cdot, \cdot)$ is coercive on $V^{0, \alpha}$, i.e., there is a constant $c_{0}$ such that

$$
c_{0}\|U\|_{V}^{2} \leq a(U, U) \quad \text { for all } U \in V^{0, \alpha} .
$$

The above conditions imply the equivalence of $(10,11)$ with (9). In the case of $\alpha=$ $(0,0,0)$, the above conditions hold provided that one restricts the spaces $V$ and $W$ to functions which are orthogonal to constants. For all other $\alpha$ in $K$, the above conditions hold with the original spaces [9].

\section{Discretization}

We will develop the approximation to (9) by the mixed approach. Stable and convergent approximations to the static problem follow provided that one uses subspaces of $V_{h} \subset V$ and $W_{h} \subset W$ satisfying discrete versions of the conditions (12) and (13). To guarantee convergence for the eigenvalue problem, additional conditions need to be verified [3]. For $\alpha=(0,0,0)$, the Nedelec approximation spaces were used for similar problems [18, 19]. To get a stable pair for $\alpha \neq(0,0,0)$, we use $\alpha$-modified spaces. The convergence and stability properties of the $\alpha$-modified spaces are given in [9]. The additional conditions which guarantee convergence for the eigenvalue problem are also verified there.

We first consider the space $V_{h}$ defined using lowest order Nedelec elements on cubes $[18,19]$. We start by partitioning the domain $\Omega$ into $N \times N \times N$ smaller cubes $\left(\Omega=\cup_{j} \Omega_{j}\right)$, each of side length $h=1 / N$. The space $V_{h}$ is defined by

$$
V_{h}=\left\{F \in H(\text { curl }):\left.F\right|_{\Omega_{j}} \in Q_{0,1,1} \times Q_{1,0,1} \times Q_{1,1,0}\right\} .
$$

Here $Q_{0,1,1}$ is the space of functions which are linear in the directions $y$ and $z$ and constant in the $x$ direction. The spaces $Q_{1,0,1}$ and $Q_{1,1,0}$ are defined analogously. The condition that $F$ be in $H($ curl) imposes continuity of the tangential components on the faces between cubes and also between faces of cubes which are translations by any index in $\Lambda$. The tangential component along the edges of the mesh of functions in $V_{h}$ are constant. In fact, any function in $V_{h}$ is uniquely determined by the values of its tangential components on the edges. One has a nodal basis $\left\{\psi_{j}\right\}$ where $\psi_{j}$ has a unit 
component tangential to the $j$ 'th edge with a vanishing tangential component along all other edges. The support of $\psi_{j}$ is contained in the cubes which contain the $j$ 'th edge. We consider here the lowest order method for simplicity. All of the techniques extend to higher order spaces in an obvious way.

The appropriate approximation space $W_{h}$ is the set of functions which are continuous and piecewise trilinear with respect to the same mesh as used in defining $V_{h}$. The spaces which result when one orthogonalizes against constants satisfy the discrete versions of (12) and (13) for $\alpha=(0,0,0)$. The usual nodal basis for $W_{h}$ shall be denoted $\left\{\phi_{j}\right\}$.

Let us consider $\alpha \neq(0,0,0)$. We need to develop spaces $V_{h}^{\alpha}$ and $W_{h}^{\alpha}$ which satisfy the condition

$$
\|w\|_{W} \leq C \sup _{\substack{X \in V_{h}^{\alpha} \\ X \neq 0}} \frac{|b(w, X)|}{\|X\|_{V}}=C \sup _{\substack{X \in V_{h}^{\alpha} \\ X \neq 0}} \frac{\left|c\left(X, \nabla_{\alpha} w\right)\right|}{\|X\|_{V}},
$$

for all $w \in W_{h}^{\alpha}$ with constant $C$ independent of $w$ and $h$. The analogous condition is proved in the case of $\alpha=(0,0,0)$ by using the fact that for any $w \in W_{h}, \nabla w \in V_{h}$. Thus, we construct spaces $\left(V_{h}^{\alpha}, W_{h}^{\alpha}\right)$ which satisfy $\nabla_{\alpha} w \in V_{h}^{\alpha}$ for all $w \in W_{h}^{\alpha}$. Although this may seem to be a difficult problem at first, it can be handled by observing that the operator $\nabla_{\alpha}$ arose in our equations $(5,6)$ from the introduction of a phase factor. This motivates the introduction of a phase factor into the definition of the mixed finite element approximation spaces. Specifically, we define

$$
\begin{aligned}
V_{h}^{\alpha} & =\operatorname{span}_{j}\left\{e^{-i \alpha \cdot\left(x-x_{j}\right)} \psi_{j}\right\}, \\
W_{h}^{\alpha} & =\operatorname{span}_{j}\left\{e^{-i \alpha \cdot\left(x-y_{j}\right)} \phi_{j}\right\} .
\end{aligned}
$$

Here $x_{j}$ is the center of the $j$ 'th edge and $y_{j}$ is the node corresponding to the nodal function $\phi_{j}$. In the above definition, $x$ is taken to vary smoothly over the support of the basis functions for degrees of freedom on the periodic boundary. It is easy to check that the above pair of spaces satisfy $\nabla_{\alpha} w \in V_{h}^{\alpha}$ for all $w \in W_{h}^{\alpha}$. The identities

$$
\nabla_{\alpha} \times\left(e^{-i \alpha \cdot\left(x-x_{j}\right)} \psi_{j}\right)=e^{-i \alpha \cdot\left(x-x_{j}\right)}\left(\nabla \times \psi_{j}\right)
$$

and

$$
\nabla_{\alpha}\left(e^{-i \alpha \cdot\left(x-y_{j}\right)} \phi_{j}\right)=e^{-i \alpha \cdot\left(x-y_{j}\right)} \nabla \phi_{j}
$$

simplify the computation of the element matrices which arise in the implementation. These spaces are no longer spaces of piecewise polynomial functions.

The approximation and stability properties for these spaces are proved in [9]. It is shown there that (14) holds and that there is a constant $c_{0}$ independent of $h$ satisfying

$$
c_{0}\|U\|_{V}^{2} \leq a(U, U) \quad \text { for all } U \in V_{h}^{0, \alpha} .
$$

Here $V_{h}^{0, \alpha}=\left\{F \in V_{h}^{\alpha}: b(g, F)=0 \quad\right.$ for all $\left.g \in W_{h}^{\alpha}\right\}$.

The approximation to problem $(10,11)$ is then: find $\omega \in \mathbb{R}$ and $\left(H_{h}, \rho_{h}\right) \in V_{h}^{\alpha} \times W_{h}^{\alpha}$ such that

$$
\begin{aligned}
a\left(H_{h}, F\right)+b\left(\rho_{h}, F\right) & =\omega^{2} c\left(H_{h}, F\right), \quad \text { for all } F \in V_{h}^{\alpha}, \\
\overline{b\left(g, H_{h}\right)} & =0, \quad \text { for all } g \in W_{h}^{\alpha} .
\end{aligned}
$$


The above problem is equivalent to: find $H_{h} \in V_{h}^{0, \alpha}$ satisfying

$$
a\left(H_{h}, F\right)=\omega^{2} c\left(H_{h}, F\right) \quad \text { for all } F \in V_{h}^{0, \alpha} .
$$

\section{Preconditioner}

Preconditioned iteration techniques for eigenvalue problems are well studied for positive definite hermitian operators $[5,10,15]$. Thus, it is most natural to develop an iterative scheme for (19). The difficulty, though, is that one does not have a computable basis for $V_{h}^{0, \alpha}$. In this section, we develop a preconditioner for the problem (19) that does not require an explicit basis for $V_{h}^{0, \alpha}$.

Given a functional $G$ on $V_{h}^{0, \alpha}$, a preconditioner involves finding the solution $D_{h} \in V_{h}^{0, \alpha}$ of the problem

$$
a_{0}\left(D_{h}, F\right)=G(F), \quad \text { for all } F \in V_{h}^{0, \alpha} .
$$

Here $a_{0}(\cdot, \cdot)$ is another positive definite Hermitian form on $V_{h}^{0, \alpha} \times V_{h}^{0, \alpha}$ that is spectrally equivalent to $a(\cdot, \cdot)$.

The way that we avoid a computational basis for $V_{h}^{0, \alpha}$ is to develop the preconditioner in mixed form. We start by defining $a_{0}(\cdot, \cdot)$ on $V \times V$ by $(7)$ with $\gamma$ set to be the constant $\gamma_{0}$ which equals the maximum of the original (spatially dependent) $\gamma$. Given a functional $G$ on $V_{h}^{0, \alpha}$, let $\widetilde{G}$ be any extension to $V_{h}^{\alpha}$. The preconditioner involves solving the problem: Find $\left(D_{h}, \eta_{h}\right) \in V_{h}^{\alpha} \times W_{h}^{\alpha}$ such that

$$
\begin{aligned}
a_{0}\left(D_{h}, F\right)+b\left(\eta_{h}, F\right) & =\widetilde{G}(F), \quad \text { for all } F \in V_{h}^{\alpha}, \\
\overline{b\left(g, D_{h}\right)} & =0, \quad \text { for all } g \in W_{h}^{\alpha} .
\end{aligned}
$$

It is easy to show that $D_{h}$ is independent of the choice of extension $\widetilde{G}$. The second equation implies that $D_{h}$ is in $V_{h}^{0, \alpha}$. Moreover, taking $F \in V_{h}^{0, \alpha}$ in the first equation shows that $D_{h}$ defined by (21) satisfies (20). Note that the solution of (21) can be computed without the use of a computational basis for $V_{h}^{0, \alpha}$.

The use of a constant coefficient in the definition of $a_{0}(\cdot, \cdot)$ enables the efficient solution of (21). It follows from this and the definition of the bases for $V_{h}^{\alpha}$ and $W_{h}^{\alpha}$ that the matrices which appear in the implementation,

$$
\begin{aligned}
& A_{j, k}^{0}=a_{0}\left(e^{-i \alpha \cdot\left(x-x_{j}\right)} \psi_{j}, e^{-i \alpha \cdot\left(x-x_{k}\right)} \psi_{k}\right), \\
& B_{j, k}=b\left(e^{-i \alpha \cdot\left(x-y_{j}\right)} \phi_{j}, e^{-i \alpha \cdot\left(x-x_{k}\right)} \psi_{k}\right)
\end{aligned}
$$

are periodically translationally invariant. Basis functions are connected (have a nonzero entry) only if the corresponding edges (or nodes) are on the boundary of some cube. The entries only depend on the geometric relation between the degrees of freedom. Clearly, there are $3 N^{3}$ edge degrees of freedom for $V_{h}^{\alpha}$ and $N^{3}$ vertex degrees of freedom for $W_{h}^{\alpha}$.

The periodically translationally invariant property means that the matrix problem corresponding to $(21)$,

$$
\left(\begin{array}{cc}
A^{0} & B^{*} \\
B & 0
\end{array}\right)\left(\begin{array}{l}
X \\
Y
\end{array}\right)=\left(\begin{array}{l}
\widetilde{g} \\
0
\end{array}\right)
$$

can be efficiently solved by application of the fast Fourier transform (FFT) (here $\widetilde{g}_{k}=$ $\left.\widetilde{G}\left(e^{-i \alpha \cdot\left(x-x_{k}\right)} \psi_{k}\right)\right)$. To see this, we group together the x-edge degrees of freedom (DOF), 
the y-edge DOF, the z-edge DOF and the vertex DOF into separate vectors. We independently apply the FFT to these four vectors and combine the results into a vector grouping together the four entries correspoding to a given Fourier component. Applying this procedure to the unknown $(X, Y)$ and the right hand side $(\widetilde{g}, 0)$ gives a vector $\widehat{Z}$ (still unknown) and a transformed right hand side $\widehat{G}$. The resulting matrix problem is

$$
\widehat{M} \widehat{Z}=\widehat{G}
$$

where $\widehat{M}$ is a block $4 \times 4$ diagonal matrix. To see this, one groups the original degrees of freedom into blocks of four where each block is associated with a mesh cube and consists of the three edge DOF corresponding to the smallest $x, y, z$ values and the corresponding vertex degree of freedom. Thus, each block has a degree of freedom for an edge parallel to the $x$-axis, $y$-axis and $z$-axis and a vertex degree of freedom. Under this reordering, the matrix

$$
\left(\begin{array}{cc}
A^{0} & B^{*} \\
B & 0
\end{array}\right)
$$

results in a matrix $M$ which has a structure consisting of block $4 \times 4$ matrices. Moreover, because of periodic translational invariance, it is completely determined by the $N^{3}$ blocks (e.g., $\left.\left\{M_{j, k}^{1, l}: l=1, \ldots, N^{3}, j, k=1,2,3,4\right\}\right)$ associated with any one of the cubes. Here the upper indices give the block location while the lower identify the entry within the block. The components in the diagonal blocks of $\widehat{M}$ are the three dimensional FFT's of the respective components in the block $4 \times 4$ matrices, i.e., $\widehat{M}_{j, k}^{l, l}=F F T\left(M_{j, k}^{1, *}\right)(l)$ where the FFT is with respect to the $*$ index. In the implementation, one preprocesses the inverses of the diagonal blocks of $\widehat{M}$. Subsequently, the computation of $D_{h}$ satisfying (21) involves 3 FFT's applied to the right hand side data $\widetilde{g}$ followed by multiplication of the inverse of the diagonal blocks followed by 3 inverse FFT's. The total work is $O\left(N^{3} \ln (N)\right)$.

\section{Subspace PRECONDitioning ALGORIthm}

The subspace preconditioning algorithm is intended to find a given number, say $s$, of the smallest eigenvalues of a large-dimensional hermitian positive definite operator. An analysis of this method and references to other similar methods can be found in $[5,15]$.

Consider the problem (19). We will give the algorithm entirely in terms of its matrix implementation. Even though the solution involves functions in $V_{h}^{0, \alpha}$, it is implemented in the basis for $V_{h}^{\alpha}$. Let $m=3 N^{3}$ be the dimension of $V_{h}^{\alpha}$. Denote by $A: C^{m} \rightarrow C^{m}$ the finite element matrix associated with the hermitian form $a(\cdot, \cdot)$ and by $C: C^{m} \rightarrow C^{m}$ the matrix associated with $c(\cdot, \cdot)$. The matrix analogue of the preconditioner is the matrix $S$ defined by $S \widetilde{g}=X$ where $X$ is the solution of (22). The subspace iteration algorithm is given as follows:

Choose an initial set of random vectors $\left\{\widetilde{g}_{i} \in C^{m}: i=1, \ldots, s\right\}$ and define $R_{0}^{\alpha}=$ $\operatorname{span}\left\{S \widetilde{g}_{1}, \ldots S \widetilde{g}_{s}\right\}$. Note that the vectors in $R_{0}^{\alpha}$ represent (are the coefficient vectors for) functions in $V_{h}^{0, \alpha}$.

For $n=0,1,2, \ldots$, perform the iteration: 
1. Compute Ritz eigenvectors $\left\{v_{j}^{n}\right\}_{j=1}^{s} \subset R_{n}^{\alpha}$, and their corresponding eigenvalues $\lambda_{1}^{n} \leq \lambda_{2}^{n} \leq \cdots \leq \lambda_{s}^{n}$ satisfying the generalized eigenvalue problem

$$
\left(A v_{j}^{n}, \phi\right)=\lambda_{j}^{n}\left(C v_{j}^{n}, \phi\right), \quad \text { for all } \phi \in R_{n}^{\alpha} .
$$

Here we use $(\cdot, \cdot)$ to denote the hermitian inner product on $C^{m}$.

2. Compute for $j=1, \ldots, s$,

$$
\hat{v}_{j}^{n+1}=v_{j}^{n}-S\left(A v_{j}^{n}-\lambda_{j}^{n} C v_{j}^{n}\right) .
$$

3. Define $R_{n+1}^{\alpha}=\operatorname{span}\left\{\hat{v}_{1}^{n+1}, \ldots, \hat{v}_{s}^{n+1}\right\}$.

The iteration (1)-(3) is terminated when $\max _{j}\left\{\left|\lambda_{j}^{n}-\lambda_{j}^{n+1}\right|\right\}$ is smaller than some prescribed tolerance. The resulting $\lambda$ 's coincide with $\omega^{2}$ in (19) up to the prescribed tolerance. Notice that the matrix-vector products $A v_{j}^{n}, C v_{j}^{n}$ are $O\left(N^{3}\right)$ operations. With $s$ fixed, one complete iteration of this algorithm is $O\left(N^{3} \ln (N)\right)$. It follows from [5] that the iteration converges at a rate which is independent of $N$.

For a full band structure calculation one generally solves a sequence of problems corresponding to a sample of points $\left\{\alpha_{m}\right\} \subset K$. The eigenvalues and eigenvectors depend continuously on $\alpha$. Consequently the eigenvectors for $\alpha_{m}$ provide a good approximation to those for $\alpha_{m+1}$ provided that $\left|\alpha_{m}-\alpha_{m+1}\right|$ is small. However, the approximation subspaces depend on $\alpha$ and and the eigenvalue iteration algorithm for requires that the initial subspace correspond to functions in $V_{h}^{0, \alpha}$ so the subspace corresponding to $\alpha_{m}$ cannot be directly used as an initial subspace for $\alpha_{m+1}$. Instead, we define an initial subspace for $\alpha_{m+1}$ as follows. Let $\left\{v_{1}^{\alpha_{m}}, \ldots, v_{s}^{\alpha_{m}}\right\}$ be the eigenvectors for $\alpha_{m}$. We define $\left\{v_{1}^{\alpha_{m+1}}, \ldots, v_{s}^{\alpha_{m+1}}\right\}$ by $v_{i}^{\alpha_{m+1}}=X$ where $X$ is the solution of $(22)$ with $\widetilde{g}=A^{0} v_{i}^{\alpha_{m}}$. Here the matrices $A^{0}$ and $B$ (appearing in (22) are defined in terms of $\alpha_{m+1}$. It is easy to show that the vectors $\left\{v_{1}^{\alpha_{m+1}}, \ldots, v_{s}^{\alpha_{m+1}}\right\}$ correspond to functions in $V_{h}^{0, \alpha_{m+1}}$ and provide no worse approximation to the eigenvectors corresponding to $\alpha_{m+1}$ than that given by $\left\{v_{1}^{\alpha_{m}}, \ldots, v_{s}^{\alpha_{m}}\right\}$. Thus, we use $\operatorname{span}\left\{v_{1}^{\alpha_{m+1}}, \ldots, v_{s}^{\alpha_{m+1}}\right\}$ as an initial subspace for the $\alpha_{m+1}$ computation. This gives good starting subspaces provided that we choose a sequence of $\alpha$ 's such that the differences $\left|\alpha_{m}-\alpha_{m+1}\right|$ are small. Proceeding in this way, one typically requires only a small number of subspace iterations for each subsequent problem after the first.

\section{NUMERICAL EXPERIMENTS}

We performed several experiments to check the method. First in the case of a homogeneous medium, we checked eigenvalues produced by the method against exact solutions. Convergence was observed as the discretization level $N$ was increased, with no spurious modes. With $N=32$, the maximum error in the first 50 eigenvalues was approximately $0.6 \%$. Next we checked the method against results in the literature obtained with the plane wave expansion method. These are illustrated in the two examples below.

Figure 2 shows a simple "scaffold" structure, similar to that analyzed by Sözüer and Haus [24]. All calculations were performed on a $16 \times 16 \times 16$ finite element grid. Figure 2(c) illustrates the band structure as $\alpha$ varies along lines connecting points of high symmetry in $K$ (shown in Figure 1). This calculation found ten eigenvalues at each of 90 values of $\alpha$ and required approximately one hour on a single processor SGI Origin 


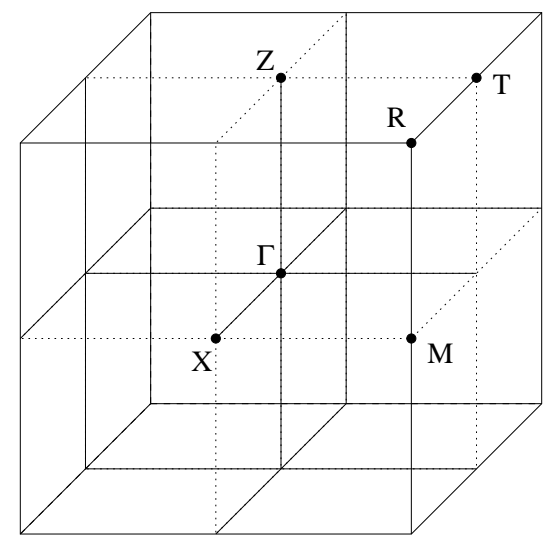

FiguRE 1. Brillouin zone $K$, showing symmetry points used for band structure plots in Figures 2(c) and 3(c).

2000. The density of states in Figure 2(d) was calculated using ten eigenvalues at 4096 uniformly spaced points in the reduced Brillouin zone.

Figure 3 shows a layered silicon structure. This structure was analyzed by Ho et al [13] and later fabricated in silicon at infrared lengthscales and analyzed by Lin et al [17]. Similar structures were analyzed by Sözüer and Dowling [23], and the idea for this type of layered structure has been attributed to unpublished work of Pendry and MacKinnon. We note that the lattice can be considered as a face-centered-cubic (fcc) primitive unit cell with a basis of two rods, however for computational simplicity we took the fundamental cell to be the $1 \times 1 \times \sqrt{2}$ rectangular solid shown in figure 3(b). The density of states figure 3(d) was calculated by computing sixteen eigenvalues at each of 4096 uniformly spaced points in the reduced Brillouin zone. The results agree well with [17], although there is a small discrepancy in the location of the gap. This can be attributed to a slight difference in bar width, which we chose at $w=0.25$ to align with our computational grid, whereas [17] used $w=0.28$.

Finally, we present the results of comparisons of our method against two plane wave methods, a Galerkin and a collocation formulation. We shall not give details of these methods here although the difference in the two methods is their treatment of $\gamma$ appearing in the form $a(\cdot, \cdot)$. These plane wave methods fall into the general framework of spectral methods and some details of their implementation can be found in, for example, $[11,21]$. Tables 1 and 2 give the first two eigenvalues as a function of the mesh size and method for the scaffold structure of Figure 2 for $\alpha=(\pi, 0,0)$. Here A denotes the mixed method described in this paper, B denotes the Galerkin plane wave approach and $\mathrm{C}$ denotes the collocation plane wave approach. It can be shown that eigenvalue approximation from Method B converge monitonically from above to the desired eigenvalue as $N$ increases. This is illustrated by the tabulated results. Method A also appears to converge in the same way. Surprisingly though, Method C appears to converge montotonically from below. Method B seems to be converging slower that the other two methods. In contrast, Method $\mathrm{C}$ appears to be converging fastest (although very little has been proved about the method). 


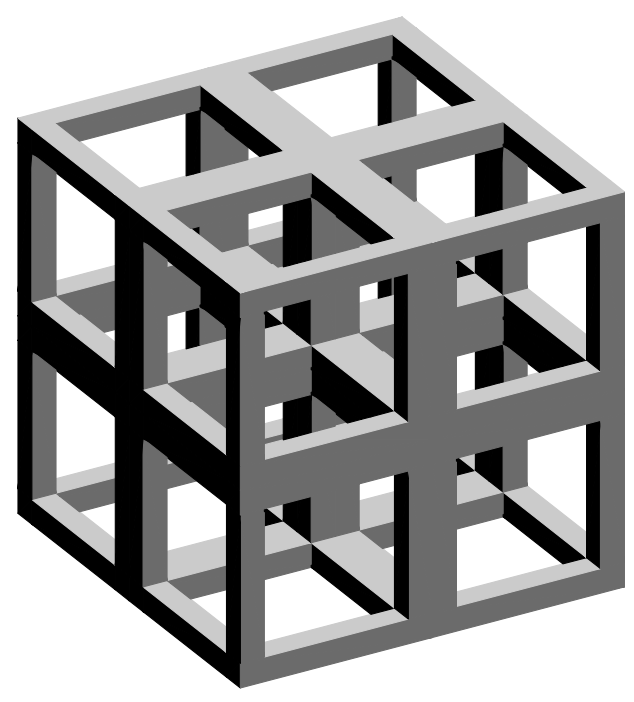

(a) Basic structure, showing $2 \times 2 \times 2$ array of cells. Refractive index is 3.6 in solid regions, 1 outside.

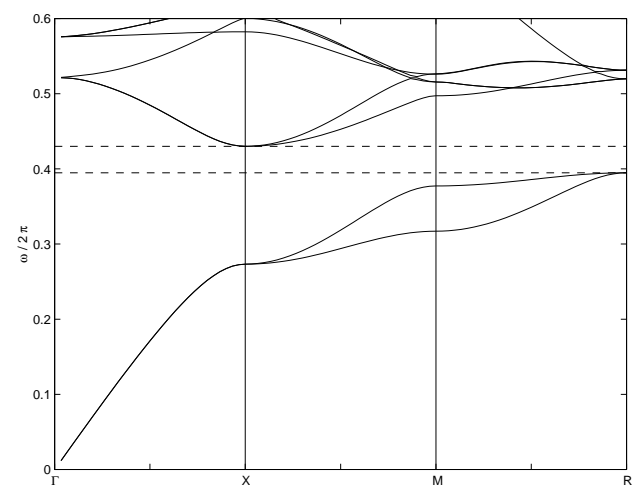

(c) Band structure. Dashed lines indicate gap edges.

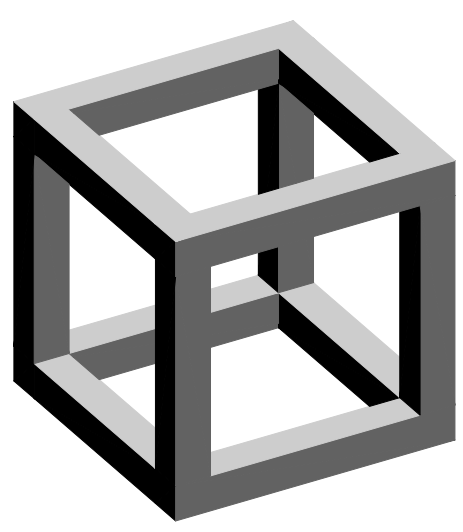

(b) Computational cell

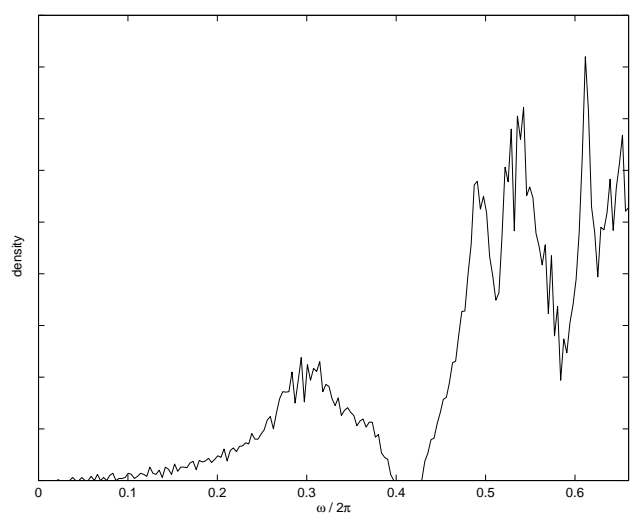

(d) Density of states.

Figure 2. Scaffold structure.

\section{Conclusion}

We have presented a new method for computing band structures in general threedimensional photonic crystals. The method combines a mixed finite element discretization, a fast Fourier transform preconditioner, and a subspace iteration algorithm to find approximate eigenvalues. The finite element discretization of the field is naturally suited to handle discontinuous media, and the subspace iteration algorithm is very efficient for computing continuously varying families of eigenvalues. Improvements in the method may be possible by investigating other eigenvalue iteration schemes or by extending the discretization scheme to allow unstructured grids. 


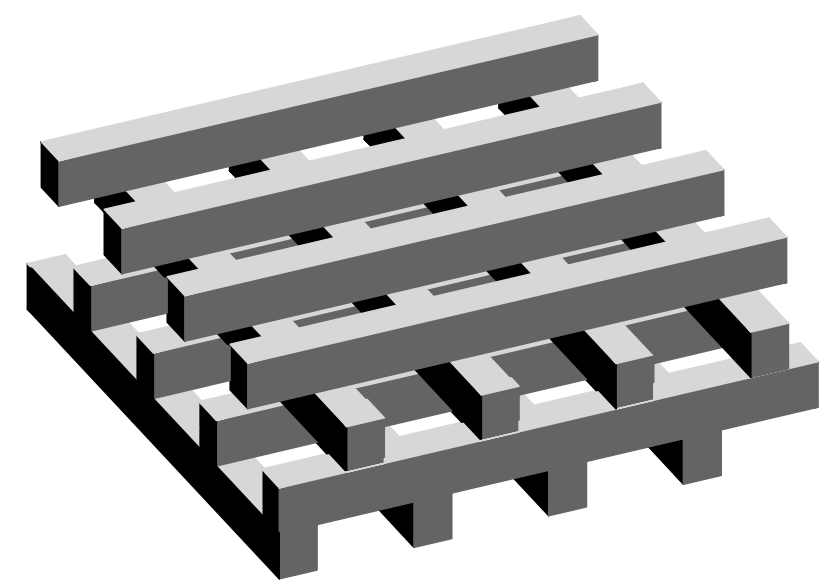

(a) Basic structure, showing one layer. Refractive index is 3.6 in bars, 1 outside.

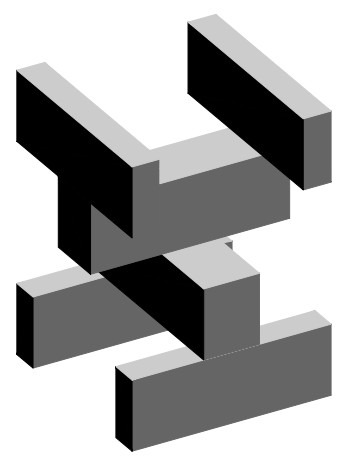

(b) Computational cell.

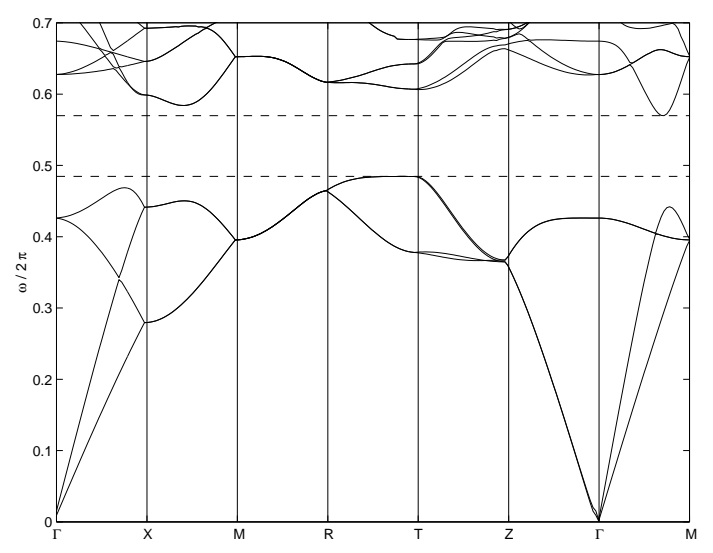

(c) Band structure. Dashed lines indicate gap edges.

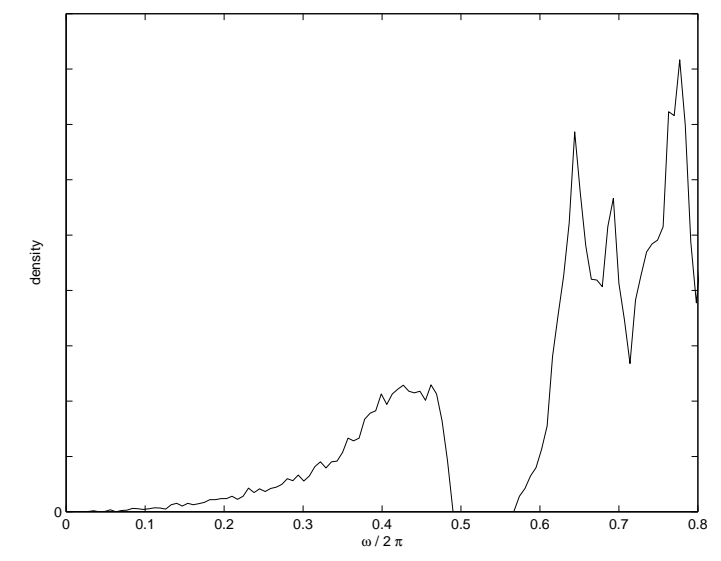

(d) Density of states.

FiguRE 3. Layered bar structure.

\begin{tabular}{|c|c|c|c|}
\hline$N$ & $\mathrm{~A}$ & $\mathrm{~B}$ & $\mathrm{C}$ \\
\hline 8 & 2.98 & 3.94 & 2.84 \\
\hline 16 & 2.95 & 3.39 & 2.90 \\
\hline 32 & 2.94 & 3.15 & 2.92 \\
\hline 64 & 2.93 & 3.04 & 2.92 \\
\hline
\end{tabular}

Table 1. First eigenvalue.

\begin{tabular}{|c|c|c|c|}
\hline$N$ & $\mathrm{~A}$ & $\mathrm{~B}$ & $\mathrm{C}$ \\
\hline 8 & 7.70 & 8.29 & 7.17 \\
\hline 16 & 7.30 & 7.74 & 7.14 \\
\hline 32 & 7.19 & 7.45 & 7.14 \\
\hline 64 & 7.16 & 7.30 & 7.14 \\
\hline
\end{tabular}

Table 2. Second eigenvalue. 


\section{ACKNOWLEDGMENTS}

The first author would like to thank P. Kuchment for many helpful discussions and ideas concerning the initial formulation of this method. We would also like to thank J. Zhao for computations involving the Galerkin plane wave method.

Acknowledgment and Disclaimer: Effort of first author sponsored by the Air Force Office of Scientific Research, Air Force Materiel Command, USAF, under grant number F49620-98-1-0005. The U.S. Government is authorized to reproduce and distribute reprints for Governmental purposes notwithstanding any copyright notation thereon. The views and conclusions contained herein are those of the authors and should not be interpreted as necessarily representing the official policies or endorsements, either expressed or implied, of the Air Force Office of Scientific Research or the U.S. Government.

\section{REFERENCES}

[1] W. Axmann and P. Kuchment, A finite element method for computing spectra of photonic and acoustic band-gap materials I. Scalar case, J. Comp. Phys. (1999).

[2] I. Babuška, On the Schwarz algorithm in the theory of differential equations of mathematical physics Tchecosl. Math. J., 8 (1958) 328-342 (in Russian).

[3] D. Boffi, F. Brezzi, and L. Gastaldi, On the convergence of eigenvalues for mixed formulations, (preprint).

[4] C. M. Bowden, J. P. Dowling, and H. O. Everitt, editors, Development and applications of materials exhibiting photonic band gaps, J. Opt. Soc. Am. B, Vol. 10, No. 2 (1993).

[5] J. H. Bramble, A. V. Knyazev, and J. E. Pasciak, A subspace preconditioning algorithm for eigenvector/eigenvalue computation, Adv. Comp. Math. 6 (1996), 159-189.

[6] F. Brezzi, On the existence, uniqueness and approximation of saddle-point problems arising from Lagrange multipliers, R.A.I.R.O., (1974), 129-151.

[7] F. Brezzi and M. Fortin, Mixed and Hybrid Finite Element Methods, Springer-Verlag, New York (1991).

[8] D. C. Dobson, An efficient method for band structure calculations in 2D photonic crystals, J. Comp. Phys. 149, (1999), 363-376.

[9] D. C. Dobson and J.E. Pasciak, Analysis of an algorithm for computing electromagnetic Bloch modes using Nedelec spaces, (in preparation).

[10] E.G. D'yakonov, Iteration methods in eigenvalue problems, Math. Notes 34 (1983) 945-953.

[11] D. Gottlieb and S.A. Orszag, Numerical Analysis of Spectral Methods: Theory and Applications, SIAM, Phil. PA, 1977.

[12] K. M. Ho, C. T. Chan and C. M. Soukoulis, Existence of a photonic gap in periodic dielectric structures, Phys. Rev. Lett. 65, No. 25 (1990), pp. 3152-3155.

[13] K. M. Ho, C. T. Chan, C. M. Soukoulis, R. Biswas and M. Sigalas, Photonic band gaps in three dimensions: new layer-by-layer periodic structures, Solid State Comm., 89 (1994), pp. 413-416.

[14] J. D. Joannopoulos, R. D. Meade, and J. N. Winn, Photonic crystals: molding the flow of light, Princeton University Press (1995).

[15] A.V. Knyazev Preconditioned eigensolvers: practical algorithms, Center Comp. Math. Rpt. \#143, Univ. Colorado at Denver.

[16] O. A. Ladyzhenskaya, The Mathematical Theory of Viscous Incompressible Flows Gordon and Breach, London, (1969).

[17] S.Y. Lin, J.G. Fleming, D.L. Hetherington, B.K. Smith, R. Biswas, K.M. Ho, M.M. Sigalas, W. Zubrzycki, S.R. Kurtz, and Jim Bur, A three-dimensional photonic crystal operating at infrared wavelengths, Nature 394 (1998), pp. 251-253.

[18] J.C. Nedelec, Mixed finite elements in $\mathbb{R}^{3}$, Numer. Math., 35 (1980) 315-341.

[19] J.C. Nedelec, A new family of mixed finite elements in $\mathbb{R}^{3}$, Numer. Math., 50 (1986) 57-81.

[20] J. B. Pendry and A. MacKinnon, Calculation of photon dispersion relations, Phys. Rev. Lett. 69, No. 19 (1992), pp. 2772-2775. 
[21] A. Quarteroni and A. Valli, Numerical Approximation of Partial Diffferential Equations SpringerVerlag, Murry Hill, NJ, 1991.

[22] M. Reed and B. Simon, Methods of Modern Mathematical Physics, Vol. IV: Analysis of Operators, Academic Press 1978.

[23] H. S. Sözüer and J. P. Dowling, Photonic band calculations for woodpile structures, J. Modern Optics, 41 (1994), pp. 231-239.

[24] H. S. Sözüer and J. W. Haus, Photonic bands: simple-cubic lattice, J. Opt. Soc. Am. B, 10 (1993), pp. 296-302.

[25] H. S. Sözüer, J. W. Haus and R. Inguva, Photonic bands: Convergence problems with the plane-wave method, Phys. Rev. B 45, No. 24 (1992), pp. 13962-13972.

Department of Mathematics, Texas A\&M University, College Station, TX 778433368 .

E-mail address: dobson@math.tamu.edu

Institute for Mathematics and its Applications, University of Minnesota, 400 Lind Hall, 207 Church Street, S.E., Minneapolis, MN 55455.

E-mail address: jayg@ima.umn.edu

Department of Mathematics, Texas A\&M University, College Station, TX 778433368 .

E-mail address: pasciak@math.tamu.edu 